Palavras chave:

Louro-pardo

Tigmomorfogênese

Regulador vegetal

Histórico:

Recebido 22/05/2015

Aceito $01 / 12 / 2015$

Keywords:

Louro-pardo

Thigmomorphogenesis

Plant growth regulator

Correspondência: danikadorin@hotmail.com
Danielle Acco Cadorin', Ubirajara Contro Malavasi', Pablo Wenderson Ribeiro Coutinho', João Alexandre Lopes Dranski', Marlene de Matos Malavasi'

\section{METIL JASMONATO E FLEXÕES CAULINARES NA RUSTIFICAÇÃO E CRESCIMENTO INICIAL DE MUDAS DE Cordia trichotoma}

RESUMO: A submissão de mudas a estímulos mecânicos e reguladores vegetais promove a sua rustificação, podendo ser incluídas na rotina dos viveiros, favorecendo a sobrevivência e o crescimento inicial a campo. O trabalho objetivou avaliar os efeitos da aplicação de metil jasmonato e de flexões caulinares na rustificação e no crescimento inicial de mudas de Cordia trichotoma. As mudas foram submetidas a 20 flexões diárias por 4 semanas e por 8 semanas; pulverização de $50 \mu \mathrm{mol} \cdot \mathrm{L}^{-1}$ de metil jasmonato aplicados semanalmente por 4 semanas e por 8 semanas e um tratamento controle. $O$ delineamento utilizado foi inteiramente ao acaso, com cinco repetições de quatorze mudas. Mudas submetidas aos tratamentos rustificativos apresentaram menor incremento em altura, maior incremento em diâmetro de coleto e menor valor para o índice de robustez. Mudas do tratamento controle tiveram maior perda de eletrólitos do tecido radicular e menor potencial de regeneração de raízes. No campo, 180 dias após o plantio, mudas submetidas a oito semanas de flexões caulinares e oito aplicações de metil jasmonato externaram maior incremento em altura e diâmetro de coleto. Os resultados indicam que tanto flexões caulinares como aplicação de metil jasmonato por oito semanas são eficientes em promover a rustificação e melhorar o desempenho inicial a campo de mudas de Cordia trichotoma.

\section{METHYL JASMONATE AND STEM BENDING HARDENING AND INITIAL GROWTH OF Cordia trichotoma SEEDLINGS}

ABSTRACT: The submission of seedlings to mechanical stimuli and plant growth regulator promote their hardening and can be included in the routine of nurseries, favoring the survival and initial growth in the field. The study aimed to evaluate the effects of applying methyl jasmonate and stem bending in hardening and initial growth of Cordia trichotoma seedlings. Seedlings were subjected to 20 stem bending daily for 4 weeks; 20 stem bending daily for 8 weeks; $50 \mu \mathrm{mol} \cdot \mathrm{L}^{-1}$ of methyl jasmonate applied weekly for 4 weeks; $50 \mu \mathrm{mol} \cdot \mathrm{L}^{-1}$ of methyl jasmonate applied weekly for 8 weeks and the control treatment. The design was a completely randomized, with five repetitions of the fourteen seedlings. Seedlings submitted to hardening treatments showed less increment in height, greater increment in stem diameter and less value for strength index. Seedlings of control treatment had greater loss of root tissue electrolytes and less potential for root regeneration. In the field, I 80 days after planting, seedlings submitted to eight weeks of stem bending and eight methyl jasmonate applications showed greater increment in height and stem diameter. The results indicate that both stem bending such as methyl jasmonate application for eight weeks are effective in promoting hardening and improve the starting performance in field of Cordia trichotoma seedlings. 


\section{INTRODUÇÃO}

A qualidade de mudas de espécies lenhosas baseia-se numa série de características morfofisiológicas que se relacionam com a sobrevivência a campo, e consequentemente com o sucesso de plantios florestais. Pesquisas indicam que algumas práticas, tais como a submissão de mudas a estímulos mecânicos e a reguladores vegetais podem estar relacionadas com a rustificação de espécies lenhosas (JACOBS; LANDIS, 2009; ORO et al., 20II; DRANSKI, 20I3; VOLKWEIS et al., 20I4). Assim, tais práticas poderiam ser incluídas na rotina dos viveiros, favorecendo a sobrevivência e o crescimento inicial de mudas a campo.

Os estímulos mecânicos têm sido realizados através de flexões caulinares em mudas. Apesar das respostas vegetais a estes estímulos serem muito variáveis entre espécies, alguns comportamentos comuns a um grande número de espécies lenhosas têm sido relatados. Entre eles estão a redução do alongamento celular, com consequente redução na altura das mudas e o aumento no diâmetro de coleto, atributos que tem servido como indicadores da qualidade de mudas (KERN et al., 2005; DRANSKI, 20I3; VOLKWEIS et al., 20I4).

A ação de reguladores vegetais pode estar relacionada à rustificação de mudas. $\mathrm{O}$ ácido jasmônico e seus derivados jasmonatos são reguladores endógenos do crescimento vegetal originários do ácido linolênico, que ocorrem em várias espécies vegetais. Esses reguladores modulam a senescência de plantas, abscisão foliar, desenvolvimento de embriões e mecanismo de defesa, agindo como sinalizadores de estresse (KERBAUY, 2008).

Cordia trichotoma (Vell.) Arrab. ex Steud, popularmente conhecida como louro-pardo, é uma espécie lenhosa pioneira nativa, de ocorrência principalmente na Floresta Estacional Semidecidual (LORENZI, 2002). A espécie apresenta potencial para reflorestamento com fins ambientais e produtivos, pois possui crescimento rápido e atinge de 20 a $30 \mathrm{~m}$ de altura com 40 a $60 \mathrm{~cm}$ de diâmetro, sendo sua madeira considerada nobre (BRACK; GRINGS, 20II).

Apesar do uso de flexões caulinares resultarem em mudas com maior rusticidade, sua execução em viveiros comercias ainda é de difícil operacionalização. Portanto, a pulverização com metil jasmonato possui caráter prático, uma vez que as respostas fisiológicas são semelhantes, e poderia substituir aquele método rustificativo.

Este ensaio objetivou avaliar os efeitos da aplicação de metil jasmonato e de flexões caulinares na rustificação e no crescimento inicial de mudas de $C$. trichotoma.

\section{MATERIAL E MÉTODOS}

Os tratamentos foram aplicados em ambiente protegido não climatizado localizado no município de Marechal Cândido Rondon, PR, durante os meses de setembro a novembro de 2014 .

O clima da região, segundo Köppen, é caracterizado como tipo $\mathrm{Cfa}$, subtropical, com temperatura média no mês mais frio inferior a 18 ${ }^{\circ} \mathrm{C}$ e temperatura média no mês mais quente acima de $22{ }^{\circ} \mathrm{C}$, com geadas pouco frequentes e tendência de concentração das chuvas nos meses de verão, contudo, sem estação seca definida. A precipitação pluviométrica anual é em torno de 1.600 a $1.800 \mathrm{~mm}$ (CAVIGLIONE et al., 2000).

Mudas de C. trichotoma foram propagadas em tubetes de $120 \mathrm{~cm}^{3}$ preenchidos com mistura de substrato comercial a base de casca de pinus e solo local na proporção de 4:I (v.v.) com incorporação de fertilizante de liberação controlada (I8N-5P2O5-9K2O) na proporção de $1,44 \mathrm{~kg} \cdot \mathrm{m}^{-3}$ de substrato. Os tubetes foram acomodados em suportes plásticos (bandejas) com capacidade de 96 tubetes e durante a fase de formação as mudas permaneceram em $50 \%$ de sombreamento.

Os valores de umidade relativa e temperatura do ar durante a aplicação dos tratamentos foram obtidos diariamente com o auxílio de um termo-higrômetro digital. A temperatura média do ambiente no momento da aplicação dos tratamentos manteve-se a $26,3^{\circ} \mathrm{C}$, e a umidade relativa do ar em $5 \mathrm{I} \%$. Durante este período as mudas permaneceram em ambiente protegido não climatizado recoberto com filme de polietileno de baixa densidade e anti-UV de 150 micra de espessura, resultando em $20 \%$ de sombreamento.

Quando as mudas apresentaram altura média de $15,0 \mathrm{~cm}$ e diâmetro de coleto de $3,22 \mathrm{~mm}$ os tratamentos foram impostos. $O$ delineamento experimental foi 0 inteiramente ao acaso com cinco repetições de quatorze mudas, que foram submetidas aos seguintes tratamentos: testemunha com água deionizada + tensoativo não iônico aplicados semanalmente; TIG4: 20 flexões diárias por 4 semanas + água deionizada e tensoativo não iônico aplicados semanalmente; TIG8: 20 flexões diárias por 8 semanas + água deionizada e tensoativo não iônico aplicados semanalmente; MJ4: $50 \mu \mathrm{mol}^{-\mathrm{L}^{-1}}$ de metil jasmonato + água deionizada + tensoativo não iônico aplicados semanalmente por 4 semanas; MJ8: $50 \mu \mathrm{mol} \cdot \mathrm{L}^{-1}$ de metil jasmonato + água deionizada + tensoativo não iônico aplicados semanalmente por 8 semanas.

A aplicação de flexões caulinares e regulador 
vegetal nos tratamentos que consistiram em apenas 4 semanas de imposição de estímulos ocorreu nas últimas semanas em relação aos tratamentos que receberam estímulos por 8 semanas. Durante as primeiras 4 semanas, as mudas que não foram tratadas permaneceram sob irrigação. Tanto na formação das mudas quanto na imposição dos tratamentos a irrigação ocorreu duas vezes ao dia até a capacidade de campo.

As flexões caulinares compreenderam estímulos mecânicos efetuados diariamente através da passagem em sentido único de uma estrutura composta por uma barra de cano de PVC com $25 \mathrm{~mm}$ de diâmetro disposta horizontalmente e fixada em uma estrutura metálica com rolamentos. As mudas foram flexionadas verticalmente não mais do que $45^{\circ}$ mediante a passagem da barra no terço inferior da folhagem, sempre no mesmo horário, a uma velocidade de $0,10 \mathrm{~m} \cdot \mathrm{seg}^{-1}$ (VOLKWEIS et al., 20I4).

A aplicação do regulador de crescimento foi realizada em intervalos de uma semana através de pulverizações foliares de metil jasmonato na concentração de $50 \mu \mathrm{mol} \cdot \mathrm{L}^{-1}$, com volume equivalente a $7 \mathrm{~mL}$ por muda, ponto em que iniciou o escorrimento foliar. As soluções foram constituídas de metil jasmonato, água deionizada e tensoativo não iônico, aplicadas com pulverizador costal manual, trabalhando a 0,3 $\mathrm{MPa}$. O tensoativo foi utilizado por melhorar a distribuição e absorção foliar do produto aplicado, através da redução da tensão superficial das gotas reduzindo o ângulo de contato destas com a superfície da folha.

Ao final do período de imposição dos tratamentos foram selecionadas ao acaso seis mudas por repetição para as determinações dos incrementos na altura da muda, no diâmetro do coleto, a relação entre altura e diâmetro do coleto (índice de robustez), as massas secas de raízes e dos tecidos aéreos, a perda de eletrólitos dos tecidos radiculares e o potencial de regeneração de raízes.

A altura da muda foi obtida com régua graduada a partir do nível do substrato até a gema apical e o diâmetro do coleto mensurado com o auxílio de um paquímetro digital. As massas secas de raízes e dos tecidos aéreos foram obtidas através de secagem em estufa de circulação de ar a $60^{\circ} \mathrm{C}$ durante $72 \mathrm{~h}$. Os incrementos foram calculados pela diferença entre os valores antes e após a imposição dos tratamentos. $O$ índice de robustez foi obtido através da razão entre a altura da parte aérea e diâmetro de coleto. $O$ teste da perda de eletrólitos dos tecidos radiculares foi realizado conforme metodologia proposta por Wilner (1955).

O potencial de regeneração de raízes foi obtido através do plantio de quatro mudas por repetição, ao final da aplicação dos tratamentos, em vasos de 3,4 $\mathrm{dm}^{3}$ preenchidos com areia. As mudas permaneceram nos vasos por 28 dias sob irrigação diária até a capacidade de campo. Após aquele período, foi quantificada a massa seca de novas raízes emergidas do torrão (LANDIS et al., 20l0).

Posteriormente a aplicação dos tratamentos rustificativos, quatro mudas por repetição foram plantadas a campo, seguindo o delineamento em blocos ao acaso, respeitando-se os tratamentos impostos durante a fase de rustificação.

O plantio ocorreu em novembro de 2014 no município de Pato Branco, PR com latitude de $26^{\circ} 13^{\prime}$ 46" S e longitude de $52^{\circ} 40^{\prime}$ ' 14" O e altitude de 760 $\mathrm{m}$. O clima da região de plantio é classificado como Cfa - clima subtropical úmido mesotérmico (IAPAR, 2000) e o solo como Latossolo Vermelho distroférrico (EMBRAPA, 2006).

Utilizou-se covas de $27 \mathrm{~cm}$ de diâmetro por $50 \mathrm{~cm}$ de profundidade em espaçamento de $3 \times 2 \mathrm{~m}$. A adubação de base constou de $100 \mathrm{~g}$ por cova da formulação $5 \mathrm{~N}-25 \mathrm{P}_{2} \mathrm{O}_{5}-25 \mathrm{~K}_{2} \mathrm{O}$. Os tratos culturais envolveram a roçada de plantas daninhas a cada 60 dias.

Aos 90 e 180 dias após o plantio foram avaliados a porcentagem de sobrevivência e os incrementos na altura e no diâmetro do coleto das mudas sobreviventes.

Os dados foram averiguados quanto à normalidade de distribuição dos resíduos pelo teste de Shapiro-Wilk e submetidos à análise da variância com auxílio do software estatístico SISVAR 5.3 (FERREIRA, 20II). Quando da existência de diferenças estatisticamente significativas, as médias foram comparadas pelo teste de Scott-Knott a $5 \%$ de probabilidade de erro.

\section{RESULTADOS E DISCUSSÃO}

O incremento na altura foi significativamente menor para mudas submetidas a flexões caulinares durante oito semanas, reduzindo em $76 \%$ a velocidade de crescimento em altura. Para mudas pulverizadas com metil jasmonato durante quatro ou oito semanas a redução foi em média de $42 \%$ em relação ao tratamento controle, e equivalendo a aplicação de flexões caulinares por quatro semanas (Tabela I). 
TABELA I Incremento na altura $(\mathrm{IH})$, no diâmetro do coleto (ID) e no índice de robustez (IR) em mudas de $C$. trichotoma submetidas a estímulos mecânicos e aplicações de metil jasmonato.

TABLE I Increment in height (IH), in stem diameter (ID) and strength index (IR) in C. trichotoma seedlings submitted to mechanical stimuli and methyl jasmonate applications.

\begin{tabular}{lccc}
\hline Tratamento & $\begin{array}{c}\mathrm{IH} \\
(\mathrm{cm})\end{array}$ & $\begin{array}{c}\text { ID } \\
(\mathrm{mm})\end{array}$ & $\begin{array}{c}\mathrm{IR} \\
\left(\mathrm{cm} \cdot \mathrm{mm}^{-1}\right)\end{array}$ \\
\hline TEST & $0,58 \mathrm{a}$ & $0,1 \mathrm{I} \mathrm{b}$ & $4,85 \mathrm{a}$ \\
TIG4 & $0,24 \mathrm{~b}$ & $0,24 \mathrm{a}$ & $4,50 \mathrm{~b}$ \\
TIG8 & $0,14 \mathrm{c}$ & $0,25 \mathrm{a}$ & $4,14 \mathrm{c}$ \\
MJA4 & $0,33 \mathrm{~b}$ & $0,22 \mathrm{a}$ & $4,52 \mathrm{~b}$ \\
MJA8 & $0,34 \mathrm{~b}$ & $0,18 \mathrm{a}$ & $4,28 \mathrm{c}$ \\
\hline CV $(\%)$ & 18,60 & 34,10 & 4,80 \\
\hline
\end{tabular}

Médias seguidas de mesma letra minúscula na coluna não diferem estatisticamente entre si pelo teste Scott-Knott a 5\% de probabilidade de erro.

Independentemente do método rustificativo aplicado por quatro ou oito semanas, observou-se um aumento médio de $102 \%$ na taxa de crescimento em diâmetro do coleto, quando comparado ao tratamento controle (Tabela I). Logo, o tratamento controle por externar maior velocidade de crescimento em altura e menor taxa de crescimento em diâmetro resultou no maior valor para o índice de robustez, assim como o menor valor foi calculado para mudas submetidas a 20 flexões caulinares por oito semanas, estando associado à redução na taxa de crescimento em altura.

Os tratamentos rustificativos resultaram em uma redução média de $22 \%$ na matéria seca dos tecidos aéreos, em comparação ao tratamento controle (Tabela 2 ), sem contudo alterar a taxa de crescimento do sistema radicular. $O$ mesmo comportamento foi detectado para a perda de eletrólitos do sistema radicular, resultando numa redução de $40 \%$.

Já para o potencial de regeneração de raízes foi observado um aumento médio 7 vezes maior em relação ao tratamento controle, independentemente do método aplicado (Tabela 2).

Por ser de fácil mensuração e um método não destrutivo, a altura da muda é amplamente utilizada para estimar o padrão de qualidade de mudas de espécies florestais, enquanto o diâmetro de coleto é de fundamental importância na avaliação do potencial da muda para sobrevivência e crescimento após o plantio (HAASE, 2008). Mudas com maior diâmetro de coleto, em geral, apresentam maior sobrevivência,
TABELA 2 Massa seca da parte aérea (MSPA), massa seca de raízes (MSR), perda de eletrólitos de raízes (PER) e potencial de regeneração de raízes (PRR) em mudas de $C$. trichotoma submetidas a estímulos mecânicos e aplicações de metil jasmonato.

TABLE 2 Shoot dry mass (MSPA), root dry mass (MSR), root electrolyte leakage (PER) and root regeneration potential (PRR) in C. trichotoma seedlings submitted to mechanical stimuli and methyl jasmonate applications.

\begin{tabular}{lcccc}
\hline Tratamento & $\begin{array}{c}\text { MSPA } \\
\left(\mathrm{g} \cdot \text { muda }^{-1}\right)\end{array}$ & $\begin{array}{c}\text { MSR } \\
\left(\mathrm{g} \cdot \text { muda }^{-1}\right)\end{array}$ & $\begin{array}{c}\text { PER } \\
(\%)\end{array}$ & $\begin{array}{c}\text { PRR } \\
\left(\mathrm{mg} \cdot \mathrm{muda}^{-1}\right)\end{array}$ \\
\hline TEST & $0,45 \mathrm{a}$ & $1,71 \mathrm{a}$ & $57,20 \mathrm{a}$ & $6,6 \mathrm{I} \mathrm{b}$ \\
TIG4 & $0,35 \mathrm{~b}$ & $1,66 \mathrm{a}$ & $31,70 \mathrm{~b}$ & $48,37 \mathrm{a}$ \\
TIG8 & $0,34 \mathrm{~b}$ & $1,81 \mathrm{a}$ & $28,70 \mathrm{~b}$ & $31,18 \mathrm{a}$ \\
MJA4 & $0,35 \mathrm{~b}$ & $1,53 \mathrm{a}$ & $43,10 \mathrm{~b}$ & $64,19 \mathrm{a}$ \\
MJA8 & $0,37 \mathrm{~b}$ & $1,64 \mathrm{a}$ & $34,90 \mathrm{~b}$ & $66,04 \mathrm{a}$ \\
\hline CV $(\%)$ & 9,20 & 15,10 & 24,40 & 22,60 \\
\hline
\end{tabular}

Médias seguidas de mesma letra minúscula na coluna não diferem estatisticamente entre si pelo teste Scott-Knott a 5\% de probabilidade de erro.

principalmente por apresentarem maior capacidade de formação de novas raízes, como reportado por Del Campo et al. (2010) em mudas de Quercus ilex L.

Estímulos mecânicos em mudas podem desencadear uma série de respostas que foram conceituadas por Jaffe (1973) como tigmomorfogênese. Menor incremento em altura e maior incremento em diâmetro de coleto têm sido alterações comumente relatadas para diversas espécies lenhosas após sua submissão à ação mecânica, devido à redução do alongamento celular (KERN et al., 2005; DRANSKI, 20I3; VOLKWEIS et al., 20l4). Tais alterações podem estar relacionadas ao fato de perturbações mecânicas abrandarem o crescimento primário e estimularem o crescimento secundário das plantas (MOREL, et al., 20l2).

Kern et al. (2005) submetendo sete híbridos de Populus trichocarpa (Torr. \& A. Gray) e Populus deltoides (Bartr. ex Marsh) a 20 flexões caulinares diárias por 80 dias, encontraram redução na altura, na biomassa da parte aérea e aumento no diâmetro de coleto para todos os híbridos em comparação com a testemunha.

As alterações resultantes no incremento em altura e diâmetro de coleto, oriundas tanto da aplicação de flexões caulinares como de metil jasmonato parecem estar relacionadas com a síntese de etileno. Biro e Jaffe (1984) afirmaram que perturbações mecânicas, tanto na forma de 
atrito como de ferimento, induzem a síntese de etileno em entrenós de Phaseolus vulgaris (L. cv. Cherokee Wax).

Trabalhos indicaram que o metil jasmonato induz a produção de etileno em diversos órgãos das plantas (FAN et al., 1998). Hudgins e Franceschi (2004) aplicaram diferentes concentrações de metil jasmonato no segundo nó de mudas de Pseudotsuga menziesii var. menziesii e encontraram indução de produção de etileno nos entrenós acima e abaixo do nó tratado.

O etileno provoca redução de crescimento de plantas por promover, além da redução no transporte de auxinas, a reorganização de microtúbulos e microfibrilas de celulose da parede celular para uma posição longitudinal, resultando em redução de altura e espessamento do caule (KERBAUY, 2008). Corroborando com o exposto, Dranski et al. (20l3) testaram a aplicação de etefon em mudas de Pachystroma longifolium (Ness). I.M. Johnst. e obtiveram redução de até $50 \%$ na altura das mudas e aumento no diâmetro de coleto de até $44 \%$ com aplicação de $600 \mathrm{mg} \cdot \mathrm{L}^{-1}$.

Já a quantificação dos íons que extravasam do tecido radicular consiste em uma forma de estimar a integridade da membrana celular das raízes. Dessa forma, quanto menor o valor observado para a perda de eletrólitos de raízes, maior a integridade na membrana da mesma (LANDIS et al., 20I0). Os menores valores encontrados para a perda de eletrólitos de raízes em mudas de $C$. trichotoma sugerem que flexões caulinares e aplicações de metil jasmonato promovem deposição de substâncias osmoticamente ativas no sistema radicular como açúcares solúveis capazes de induzir tolerância a diversos estresses ambientais (GUO et al., 20l0).

O potencial de regeneração de raízes foi significativamente menor para o tratamento controle, indicando que mudas submetidas aos diferentes métodos rustificativos tiveram a produção de novas raízes estimuladas. $O$ teste de potencial de regeneração de raízes é preditivo do desempenho da muda a campo, e tem sido um bom indicador de qualidade de mudas, uma vez que seu resultado é a expressão de vários parâmetros fisiológicos, que culminam na habilidade de uma muda iniciar e alongar novas raízes em um dado período de tempo e condição ambiental tida como ótima ao crescimento (DAVIS; JACOBS, 2005; LANDIS et al., 2010). Desta forma, a capacidade de crescimento de novas raízes é determinante para que ocorra a conexão entre o sistema radicular da muda com o solo do local de plantio (GROSSNICKLE, 20I2).

Portanto, ao final da fase de viveiro fica evidente que a aplicação de 20 flexões caulinares e a pulverização de metil jasmonato na concentração de $50 \mu \mathrm{mol} \cdot \mathrm{L}^{-1}$ possibilita obter mudas com maior rusticidade, sendo o crescimento aéreo mais afetado com oito aplicações semanais.

Os métodos rustificativos aplicados não resultaram em maior sobrevivência das mudas em campo, tanto aos 90 como aos 180 dias do plantio. A porcentagem de sobrevivência foi de $97 \%$ e $95 \%$ aos 90 e 180 dias, respectivamente.

Os resultados obtidos após 90 dias do plantio indicaram ausência de significância para a taxa de crescimento em altura. Já para a taxa de crescimento em diâmetro do coleto, mudas submetidas a 20 flexões caulinares diárias durante oito semanas externaram maior incremento, resultando em um aumento de 107\% em relação ao tratamento controle (Tabela 3).

Contudo, após 180 dias do plantio as taxas de crescimento em altura e no diâmetro do coleto foram potencializadas quando da aplicação de 20 flexões caulinares diárias ou da pulverização de metil jasmonato por oito semanas, resultando no aumento da velocidade de crescimento em altura e no diâmetro do coleto de $130 \%$ e 102\%, respectivamente, em comparação ao tratamento controle.

TABELA 3 Incremento na altura $(\mathrm{IH})$ e no diâmetro do coleto (ID) em mudas de $C$. trichotoma submetidas a estímulos mecânicos e aplicações de metil jasmonato após 90 e 180 dias de plantio a campo.

TABLE 3 Increment in height (IH) and in stem diameter (ID) in $C$. trichotoma seedlings subjected to mechanical stimuli and methyl jasmonate applications after 90 e 180 days of out planting.

\begin{tabular}{lcccc}
\hline Tratamento & $\mathrm{IH}(\mathrm{cm})$ & $\mathrm{ID}(\mathrm{mm})$ & $\mathrm{IH}(\mathrm{cm})$ & $\mathrm{ID}(\mathrm{mm})$ \\
\cline { 2 - 5 } & \multicolumn{2}{c}{$(90$ dias$)$} & \multicolumn{2}{c}{$(\mathrm{I} 80$ dias $)$} \\
\hline TEST & $23,87 \mathrm{a}$ & $2,89 \mathrm{~b}$ & $25,8 \mathrm{~b} \mathrm{~b}$ & $4,52 \mathrm{~b}$ \\
TIG4 & $22,93 \mathrm{a}$ & $3,25 \mathrm{~b}$ & $39,68 \mathrm{~b}$ & $5,83 \mathrm{~b}$ \\
TIG8 & $39,09 \mathrm{a}$ & $5,98 \mathrm{a}$ & $62,05 \mathrm{a}$ & $8,84 \mathrm{a}$ \\
MJA4 & $28,65 \mathrm{a}$ & $3,95 \mathrm{~b}$ & $41,50 \mathrm{~b}$ & $6,36 \mathrm{~b}$ \\
MJA8 & $23,78 \mathrm{a}$ & $4,15 \mathrm{~b}$ & $56,80 \mathrm{a}$ & $9,34 \mathrm{a}$ \\
\hline C.V. (\%) & 30,00 & 30,40 & 24,90 & 28,30
\end{tabular}

Médias seguidas de mesma letra minúscula na coluna não diferem estatisticamente entre si pelo teste Scott-Knott a 5\% de probabilidade de erro.

Aos 90 dias após o plantio a campo Dranski (20l3) encontrou $25 \%$ de incremento em altura em mudas de Pinus taeda L. submetidas a 35 flexões diárias por trinta dias, e aumento de $28,2 \%$ no diâmetro de coleto em mudas tratadas com 23 flexões caulinares em relação ao tratamento controle, indicando que a imposição do tratamento na fase de viveiro potencializou 
- crescimento inicial de mudas a campo, o que corrobora com os resultados encontrados no presente estudo.

Os maiores incrementos observados na altura e no diâmetro de coleto em mudas tratadas com flexões caulinares e metil jasmonato durante oito semanas sugerem que o sucesso dos plantios florestais depende, dentre outras variáveis, da qualidade de mudas utilizadas na implantação do povoamento florestal, que por sua vez está relacionada com características morfofisiológicas das mesmas.

Dessa forma, os resultados indicaram que as alterações morfofisiológicas resultantes das flexões caulinares e da aplicação do regulador vegetal por oito semanas estão relacionadas com o melhor desempenho inicial das mudas a campo, e, portanto, a pulverização com metil jasmonato poderá substituir aplicação das flexões caulinares no viveiro, uma vez que as respostas fisiológicas evidenciadas nos tratamentos assemelham-se entre si.

\section{CONCLUSÕES}

Aplicações de flexões caulinares ou metil jasmonato por oito semanas são eficientes em promover a rustificação e melhorar o desempenho inicial a campo de mudas de Cordia trichotoma.

\section{AGRADECIMENTOS}

Externamos agradecimentos à CAPES, CNPq e FUNDAÇÃO ARAUCÁRIA pela concessão de bolsas de estudo.

\section{REFERÊNCIAS}

BIRO, R.; JAFFE, M.J. Thigmomorphogensis: ethylene evolution and its role in the changes observed in mechanically perturbed bean plants. Physiologia Plantarum, v.62, n.2, p.289-296, 1984.

BRACK, P.; GRINGS, M. Espécies nativas da flora brasileira de valor econômico atual ou potencial: plantas para o futuro - Região Sul. Brasília, MMA, 20II. 936p.

CAVIGLIONE, J.H.; KIIHL, L.R.B.; CARAMORI, P.H.; OLIVEIRA, D. Cartas climáticas do Paraná. Londrina: IAPAR, 2000. CD-ROM.

DAVIS, A.S.; JACOBS, D.F. Quantifying root system quality of nursery seedlings and relationship to out planting performance. New Forests, v.30, n.2-3, p.295-3। I, 2005.
DEL CAMPO, A.D.; NAVARRO, R.M.; CEACERO, E.C.J. Seedling quality and field performance of commercial stocklots of containerized holm oak (Quercus ilex) in Mediterranean Spain: an approach for establishing a quality standard. New Forests, v.39, n. I, p. I9-37, 2010.

DRANSKI, J.A.L.; MALAVASI, U.C.; MALAVASI, M.M.; JACOBS, D.F. Effect of ethephon on hardening of Pachystroma longifolium seedlings. Revista Árvore, v.37, n.3, p.40I-407, 2013

DRANSKI, J.L. Tigmomorfogênese na rustificação e sobrevivência em mudas de Pinus taeda L. 2013. 107p. Tese (Doutorado em Agronomia) - Universidade Estadual do Oeste do Paraná, Marechal Cândido Rondon.

EMPRESA BRASILEIRA DE PESQUISA AGROPECUÁRIA EMBRAPA. Centro Nacional de Pesquisa de Solos. Sistema brasileiro de classificação de solos. Brasília, 2006. 306p.

FAN, X; MATHEIS, J. P; FELLMAN, J.K. A role for jasmonates in climacteric fruitripening. Planta, v.204, p.444-449, 1998.

FERREIRA, D.F. SISVAR: a computer statistical analysis system. Ciência e Agrotecnologia, v.35, n.6, p. 1039-1042, 201 I.

GROSSNICKLE, S.C. Why seedlings survive: influence of plant attributes. New Forests, v.43, n.5-6, p.7I I-738, 20 I 2.

GUO, J.; YANGA, Y.; WANGA, G.; YANGA, L.; SUNA, W. Ecophysiological responses of Abiesfabri seedlings to drought stress and nitrogen supply. Physiologia Plantarum, v.139, n.4, p.335-347, 2010.

HAASE, D.L. Understanding forest seedling quality: measurements and interpretation. Tree Planters Notes, v.52, n.2, p.24-30, 2008

HUDGINS, J.W.; FRANCESCHI, R.V. Methyl jasmonateinduced ethylene production is responsible for conifer phloem defense responses and reprogramming of stem cambial zone for traumatic resin duct formation. Plant Physiology, v. I35, p.2134-2149,2004.

IAPAR. INSTITUTO AGRONÔMICO DO PARANÁ. Cartas climáticas do Paraná. Londrina: IAPAR, 2000. CD-ROM.

JACOBS, D.F;; LANDIS, T.D. Hardening. In: DUMROESE, R.K.; LUNA, T.; LANDIS, T.D. (Eds.). Nursery manual for native plants: Guide for tribal nurseries. v.I. Washington: United States Department of Agriculture, Forest Service, 2009. p.217-228.

JAFFE, M. J. Thigmomorphogenesis: the response of plant growth and development to mechanical stimulation with special reference to Bryoniadioica. Planta, v.lI4, n.2, p.143-I56, 1973.

KERBAUY, G.B. Fisiologia Vegetal. Rio de Janeiro: Guanabara Koogan, 2008. 
KERN, A.K.; WERS, W.F.; TELEWSKI, W.F.; KOEHLER, L. Mechanical perturbation affects conductivity, mechanical propertiesand aboveground biomass of hybrid poplars. Tree Physiology, v.25, n. 10, p.1243-I25I, 2005.

LANDIS, T.D.; DUMROESE, R.K.; HAASE, D.L. The container tree nursery manual: seedling processing, storage, and out planting. v.7. Washington: Department of Agriculture Forest Service, 2010.

LORENZI, H. Árvores brasileiras: manual de identificação e cultivo de plantas arbóreas nativas do Brasil. 4. ed. Nova Odessa: Instituto Plantarum, 2002. v. I. 384p.

MOREL, P., CRESPEL, L., GALOPINC, G., MOULIAD, B. Effect of mechanical stimulation on the growth and branching of garden rose. Scientia Horticulturae, v. I35, p.59-64, 2012.
ORO, P.; VOLKWEIS, R.C.; NEIVERTH W.; DRANSKI, J.A.L.; MALAVASI, U.C.; MALAVASI, M.M. Aplicação de regulador vegetal na aclimatação de mudas de Cariniana estrellensi.Cultivando o Saber, v.5, n.4, p.|03-II2, 201 I.

VOLKWEIS, R.C.; DRANSKI, J.A.L.; ORO, P.; MALAVASI, U.C.; MALAVASI, M.M. Efeito da tigmomorfogênese na morfometria de mudas de Maytenus ilicifolia (Schrad.) Planch. Ciência Florestal, v.24, n.2, p.339342, 2014.

WILNER, J. Results of laboratory tests for winter hardiness of woody plants by electrolyte methods. Proceedings American Horticultura Science, v.66, p.93-99, 1955. 
